\title{
The application of intelligent decision support technology in distance education system
}

\author{
QIAO Zhu Feng ${ }^{1,2, a}$, GUO Jian Xin ${ }^{1,2, b}$ and ZHAO Ji Chun ${ }^{1,2, c}$
}

${ }^{1}$ Institute of Information on Science and Technology of Agriculture, Beijing Academy of agriculture and forestry Sciences, Beijing, China

${ }^{2}$ The Research Center of Beijing Engineering technology for Rural Remote Information Services, Beijing, China

aqiaozf@agri.ac.cn, bguojx@agri.ac.cn, ${ }^{\mathrm{c} z h a o j c @ a g r i . a c . c n ~}$

Keywords: Distance Education; Intelligent; Decision Support; Data Warehouse; Assessment

Abstract. This paper analyzes the current situation and existing problems of the statistical analysis of the distance education system, the design and evaluation of the overall framework of the intelligent decision support system are described in detail, and the statistical analysis and decision analysis are made of the three subsystems. The realization of data warehouse, knowledge base and data mining technology in the decision support system of distance education platform is described in detail.

\section{Introduction}

With the development of the times, change rapidly. The development of information technology has brought about great changes in people's way of life. In recent years, network information technology, mobile Internet, big data technology is a hitherto unknown speed around us. From the Internet to the mobile Internet to the development of big data technology. The Internet field and application has been greatly extended. At the same time, the data expansion has opened another era. The era of big data with the curtain opened, the rapid development of computer and network, quantization, for large data collection, analysis, processing technology makes people's understanding of big data further. Individuals can also better from structured data, refine the target information, the unstructured data. Therefore, data on the political, economic, cultural, and other aspects of life have brought innovation and challenge. In this variable in leather, the education industry is also deeply affected, whether it is education or work mode of management, as well as education and learning behavior and teaching management assessment and so on because of big data and have new opportunities.

\section{System Structure}

The distance education decision support system includes the assessment system, the statistical system and the decision analysis system. Among them, the assessment system includes evaluation index system management, curriculum organization assessment, training resource assessment and teaching management assessment; statistical system including training situation real-time statistics, training organization statistics, learning hot spot statistics and customized statistical reports; decision analysis system including data analysis and development trend analysis. The system function structure diagram is shown in Figure 1.

\section{Assessment System}

Assessment system is mainly to achieve the evaluation index system of management, program evaluation organization, evaluation of training resources, teaching management assessment, etc., for individual autonomous learning provide a reference to provide the basis for the optimal allocation of teaching resources, for evaluation of the organization department management to provide reference data. 
Evaluation index system is used to define how to assess teaching, learning, curriculum content, the administrator team, the index system will be teaching and learning situation, etc., to carry out a comprehensive calculation, to complete the final assessment. Curriculum evaluation is the evaluation of the organization of the curriculum when users are learning. Training resources assessment is the training of resources for scoring, comprehensive assessment of the training resources of the scores.

The comprehensive evaluation of teaching management is aimed at the teaching of each item, according to the teaching evaluation results of the project, learning effect evaluation results, the preparation of the situation before the teaching management issues, comprehensive evaluation of the project's teaching management scores.

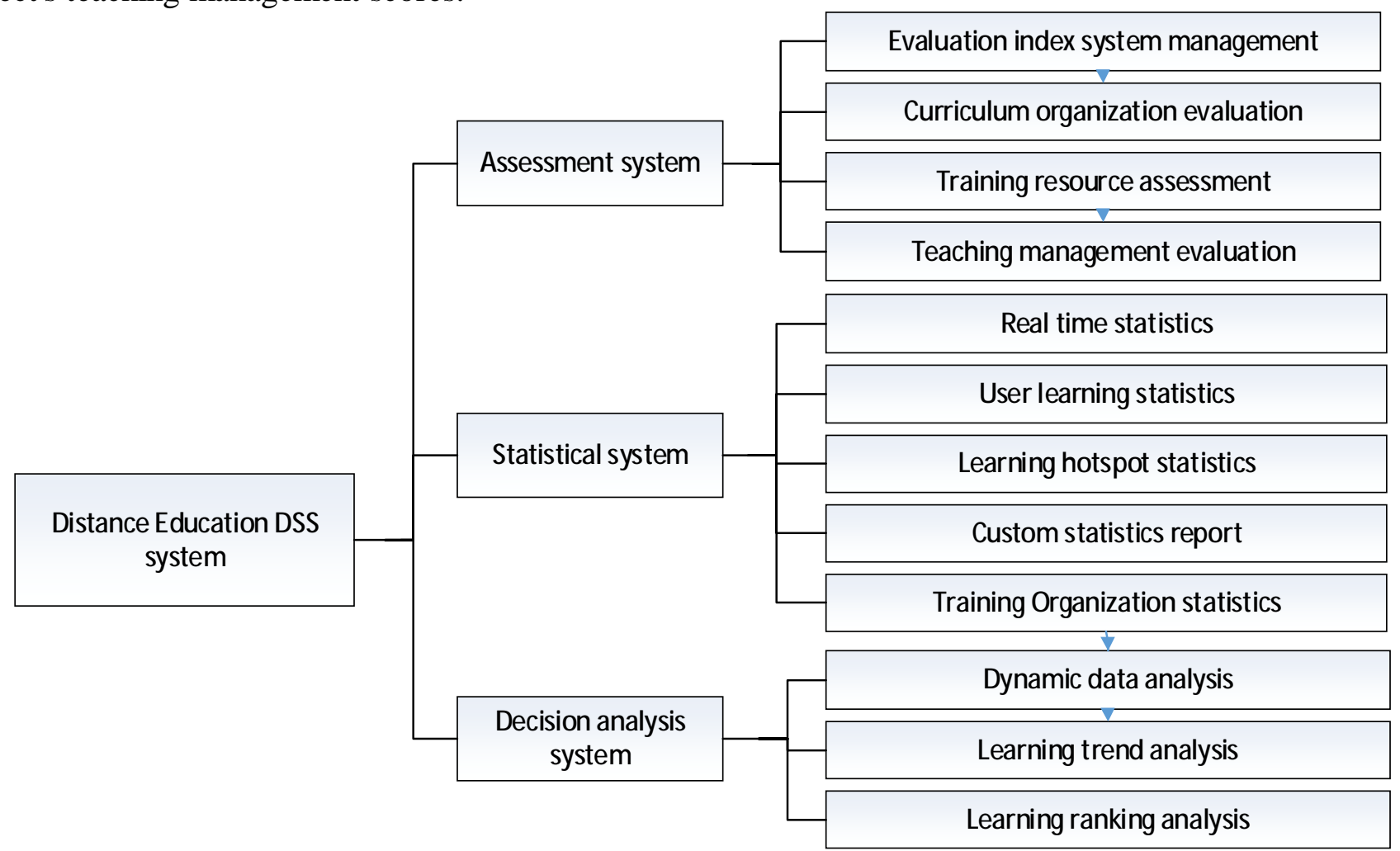

Figure 1: System function structure diagram

\section{Statistical System}

The statistical system mainly realizes the long-distance education situation real-time statistics, the Remote Education Station Statistics, the distance education organization statistics, the study hot spot statistics, the custom statistics report forms and so on, the combination classification management can provide the different statistical analysis service for each level organization.

Distance education situation real-time statistical function statistical user training project selection situation, the training project attendance, training project completion, the total number of training projects, the total attendance of training programs, training project completion rate and so on.

Distance Education Organization statistics user training of the relevant registration, training programs to complete the total number of people and so on. Learning hot statistical functions of statistical hot courses, the choice of user programs. Custom statistical report forms the overall application function to support the visualization of statistical report management applications, operators can be customized according to the requirements of the content of the report, and then ensure the comprehensive display of the report.

The site information statistics can statistics party members and cadres of modern distance education infrastructure, teaching resources, the backbone of the team, teaching organization and other work information, timely grasp of the use of the terminal site and resource information application, convenient statistics site usage, courseware on demand rate, feedback the demand and 
satisfaction of rural Party members and cadres, fully reflect the network system of distance education work dynamic, for the leadership decision-making services.

\section{Decision Analysis System}

Decision analysis system includes remote education dynamic data analysis, distance education decision support two parts, based on data warehouse technology of massive data for multidimensional analysis and processing, to achieve the training of municipal distance education platform to carry out, the use of resources and departments teaching resources quality data analysis and trend analysis, for the development of Distance Education provide auxiliary decision support. The user can according to the actual needs of the work of Party building and management work in distance education and customized decision analysis model, through to municipal platform massive historical data of flexible analysis for the future layout of the work and policy formulation to provide decision support.

This includes all kinds of education and training of the relevant data, the use of resources related to the data, the relevant data of the quality of teaching resources, in accordance with the intuitive three-dimensional columnar graphics and graphics display, greatly enhance the analysis of massive data processing capacity.

Through the education and training of the situation, the use of resources, the Department of teaching resource quality analysis, the important indicators for monitoring, to provide decision-making support for the decision makers, and the future development direction and operation of the trend to make scientific prediction, the development trend analysis

\section{System Implementation}

The system is divided into 4 layers structure, data preprocessing, the source data layer, data preprocessing. Layer, data mining analysis layer and presentation layer. The data in the source data layer is analyzed and processed, and then it is loaded into the data warehouse, using online analytical processing and data mining technology in the data analysis layer, combined with the knowledge rules of intelligent knowledge base, the data is analyzed and evaluated, the data is converted to decision information, and then through the data interface to show the results to the decision makers, to assist the decision makers to make the right decision.

The framework of the intelligent decision support system is shown in Figure 2.

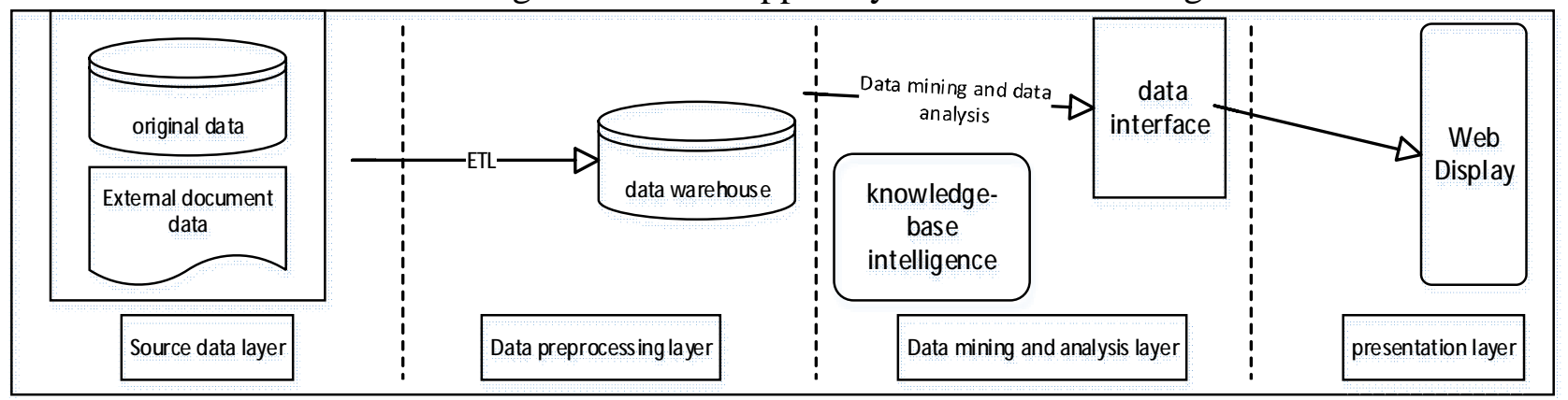

Figure 2: System technical architecture

The data warehouse model is built by extracting and transforming the data from other systems, and a variety of analysis modules are established based on the data warehouse model. 1) Through the data extraction script, the need of the system database of the new data and the possible change of data extraction to the database of the decision support system, the main need to extract data from the following database: village official online database, red harbor database, the mass line database, portal database, mobile platform database. 2) After extraction, according to the various functional modules, statistical calculation of relevant data, mainly divided into real-time online, site use, training and learning, interactive learning, hot spot analysis, hot topics, user distribution, etc. 


\section{Construction of Data Warehouse}

Construction of data warehouse of distance education platform, data consistency is the trend of distance education information technology, data warehouse provides a strong support, data warehouse according to the theme of the form to organize data, remote education portal, remote education streaming media management system, distance education platform system and mobile learning platform for remote education platform decision support.

\section{Construction of Intelligent Knowledge base}

Build intelligent knowledge base, improve the problem solving ability. Knowledge acquisition is the foundation of knowledge base, knowledge can be obtained by manual and automatic way. Some expert experience and research data can be sorted into knowledge base, relevant indicators and other contents can be manually sorted into the standard library, and finally the knowledge base, database, knowledge through data mining, machine learning to find knowledge, perfect knowledge base.

\section{Online Analytical Processing and Data Mining}

The use of intelligent analysis technology to strengthen the level of data analysis through online analysis processing technology, remote education platform database is difficult to understand the data analysis, it is easy to understand and apply the regularity of information. Data mining technology, highly automated analysis of the distance education platform for data, to make inductive reasoning, extract potential, valuable information from the massive data, so as to support decision support.

\section{Conclusions}

Through the application of the system can be verified, the decision support system can accelerate the pace of information technology in distance education platform, effectively improve the working efficiency of decision makers. With the maturity of the big data technology, the improvement of the level of big data applications, the implementation of the decision support system in the distance education platform will have a better future.

\section{References}

[1] Deng Wenjun, Cao Yangbo, Liu Yingqun, Cheng Jiangang:Research and design of data analysis tool based on educational administration management system. Learning Resource and Technology China Educational Technology Vol328,2014.05.

[2] Xia Huosong Zhen Huachun: public opinion analysis and decision support study under big data surroundings. journal of intelligence Vol.34 No.2,Feb.2015.

[3] Agrawal D,Budakc,El Abbadi A,et al.Big data in Online Social Network:UserBehavior in Social Networks[M].Database in Networked Information Systems.Springer International Publishing,2014:1-16.

[4] Li Haibo, Cheng Yaodong. Large data storage technology and standardization. Information technology and standardization, 2013 (5).

[5] Xu Peng, Wang Yining, Liu Yanhua, Zhang Hai. Analysis and study from the perspective of big data change -- Report on the interpretation and Revelation of 《through education data mining and learning analysis to promote teaching and learning 》[J]. Journal of distance education, 2013 (6): 11-17. 